\title{
Design of Data Acquisition System for Green Laser Photoacoustic Spectroscopy Using Audacity Software
}

\author{
Frida Agung Rakhmadi ${ }^{1}$, Mitrayana ${ }^{2}$, Gilang Kurniawan Dian Pangestu ${ }^{3}$ \\ ${ }^{1}$ Physics Department, Faculty of Science and Technology, UIN Sunan Kalijaga \\ ${ }^{2}$ Physics Department, Faculty of Mathematics and Natural Sciences, UGM, ${ }^{3}$ PT Unindo Hi-Tech Pratama \\ Email: frida.rakhmadi@uin-suka.ac.id ${ }^{1}$, mitrayana@ugm.ac.id ${ }^{2}$, gilang.mkt@unindopratama.com
}

\begin{abstract}
Research on design of data acquisition system for green laser photoacoustic spectroscopy has been done. The purpose of this research was to create and test data acquisition system for green laser photoacoustic spectroscopy. The system was created with ATR 3350iS microphone as signal detector and audacity software as data recording and processing. The testing was conducted in four phases, firstly testing the system without laser, secondly testing the system with non-modulated laser, thirdly testing the system with modulated laser without a sample, and finally testing the system with modulated laser to wood sample. The result showed that there weren not any photoacoustic signals detected in condition of without laser and with non-modulated laser. Meanwhile, in the condition of modulated laser without sample and with the sample of wood, the data acquisition system detected photoacoustic signals in the range of laser modulation frequency was $1000-9981 \mathrm{~Hz}$.
\end{abstract}

Keywords: ATR 3350iS microphone, Audacity software, Data acquisition system, Green laser photoacoustic spectroscopy

\section{INTRODUCTION}

Spectroscopy is one technology that plays an important role in the world of material analysis (Rosencwaig, 1975; Rocenwaig and Gersho, 1975). One spectroscopy that is currently developing is photoacoustic spectroscopy (Amin and Mitrayana, 2013), among them using lasers as the source of light (Bageshwar et al., 2010).

Laser photoacoustic spectroscopy is a type of spectroscopy that utilizes laser light to produce sound. If a material is exposed to laser light, there will be energy conversion from radiation energy to sound energy.

A system of laser photoacoustic spectroscopy consists of a laser modulation sub-system and a data acquisition sub-system. The sub-system of laser modulation functioned to modulate the laser light. In the laser photoacoustic spectroscopy, the laser light is not converted to sound when the laser not modulated firstly. Meanwhile, the data acquisition sub-system is useful for detecting sounds, processing them, and storing the processed data.

The development of current technologies has opened opportunities for research in laser photoacoustic spectroscopy instruments. The presence of a green laser has been implemented by Rakhmadi and friends in the research on design of a green laser modulation system. The result of research has been published in AIP Conference Proceedings 2014 (020149-1 - 020149-5).

In addition to hardware progress, software progress also did not lose. The presence of software that was easier and more practical, one of them is Audacity, has opened the opportunity for research in manufacturing of green laser photoacoustic spectroscopy data acquisition systems using Audacity. Beside of its easy and practical, Audacity is an open source software.

\section{MATERIALS AND METHODS}

The research on design of data acquisition system using Audacity software was done in two phases: manufacturing and testing of data acquisition system. The two phases above are described as follows.

Manufacturing of data acquisition system consists of two phases: making system and connecting with computer. The microphone used was ATR 3350iS. This microphone was chosen because its range was wide enough in audible area. In addition, this microphone also has a small design that made it easy to adjust system layout and also compatible with computer that used without need of a converter from other devices. The data acquisition system that has been manufactured then connected to the software on computer by connecting the microphone output to the computer's lay-in input.

Testing of data acquisition system of green laser photoacoustic spectroscopy consists of testing with a modulated laser without sample and with samples. Testing of data acquisition system with modulated laser aimed to determine the range of data acquisition system in detecting photoacoustic signals. This test was done by recording the sound detected by the microphone. The laser modulation frequency varies from $500 \mathrm{~Hz}$ to $10,000 \mathrm{~Hz}$ at intervals of $500 \mathrm{~Hz}$. Each frequency of laser modulation was repeated 5 times. Meanwhile, testing the data acquisition system on sample with modulated laser, the sample used was a wood. The procedure was same as testing procedure of data 
acquisition system with modulated laser without sample.

The testing results of laser modulation system, both in the absence of sample and in the presence of sample, were analyzed by audacity analysis and system analysis. Audacity analysis aimed to evaluate the recording results of each test on the frequency domain. This analysis was done by applying the principle of Fast Fourier Transform (FFT). Meanwhile, system analysis was carried out to evaluate the range of data acquisition system that has been made for detecting photoacoustic signals.

\section{RESULTS AND DISCUSSION}

\section{Manufacturing of Data Acquisition System}

The data acquisition system using audacity software that has been manufactured in this research was presented in (Figure 1).

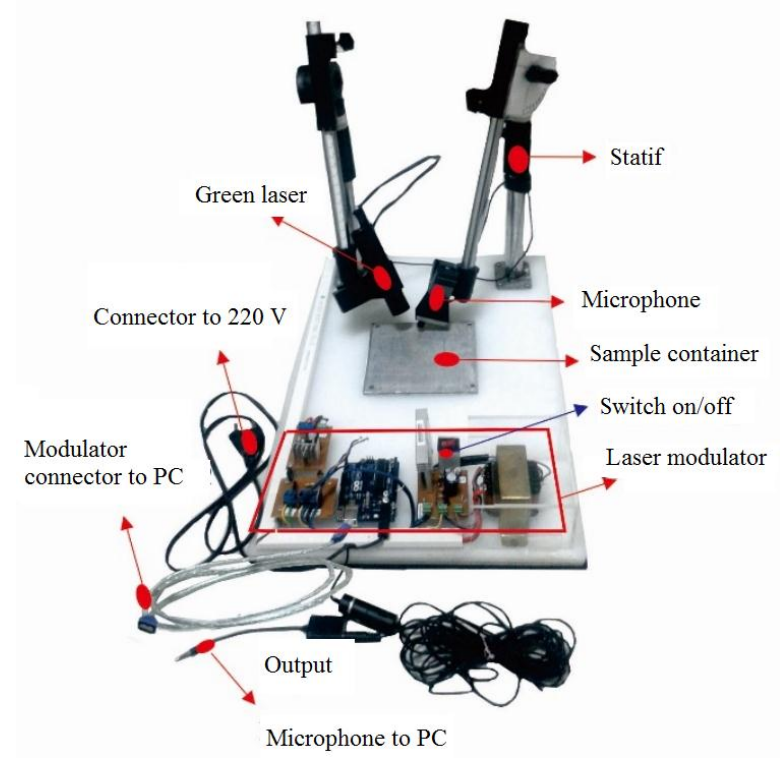

Figure 1. The data acquisition system of green laser photoacoustic spectroscopy.

The system work in (Figure 1) begins by activating the system (pressing the on-button). After the system is active, then the frequency and cicle duty are adjusted to the laser modulation system. This arrangement is done by inputting a value corresponding to the frequency and duty cicle according to the desired output of the laser modulation system.

Such settings will result in an output green laser has a code, with frequency and cycle duty values that correspond to the output of the laser modulation system. The effect is a phenomenon called photoacoustic signal.

The photoacoustic phenomenon was then recorded by a microphone with Audacity software. From recorded data, to find out the detail of photoacoustic peak signal (in the form of amplitude ( $\mathrm{dB}$ ) and the frequency of the acoustic signal), a spectrum analysis process was carried out by changing the recorded data from time domain to frequency domain. The process was found on menu in Audacity, so that analysis process, user can directly select spectrum plot section in analyzing sub-menu.

\section{Testing of Data Acquisition System}

Comparison of acoustic amplitude sin the test without samples and with samples is shown in Table 1.

Table 1. Acoustic amplitudes at many frequencies of laser modulation system.

\begin{tabular}{llll}
\hline $\mathbf{f}(\mathbf{H z})$ & \multicolumn{2}{c}{$\bar{A} \pm \Delta A \mathbf{( d B )}$} & Note \\
\cline { 2 - 3 } & Without & With & \\
\hline 500 & - & - & \\
1000 & $-76,8 \pm 0,4$ & $-76,1 \pm 0,2$ & Different \\
& $-80,1 \pm 0,2$ & & Different \\
& $-82,0 \pm 0,3$ & $-83,6 \pm 0,3$ & Different \\
& $-83,9 \pm 0,3$ & $-84,9 \pm 0,3$ & Different \\
& $-85,5 \pm 0,8$ & $-87,4 \pm 0,2$ & Different \\
1500 & $-84,08 \pm 0,3$ & $-83,6 \pm 0,2$ & Same \\
& $-86,34 \pm 0,3$ & $-86,0 \pm 0,3$ & Same \\
2000 & $-75,6 \pm 0,1$ & $-75,7 \pm 0,1$ & Same \\
& $-83,8 \pm 0,3$ & $-84,4 \pm 0,2$ & Same \\
& $-86,9 \pm 0,4$ & $-87,5 \pm 0,3$ & Different \\
2505 & $-84,2 \pm 0,2$ & $-85,02 \pm 0,08$ & Different \\
3012 & $-72,3 \pm 0,2$ & $-71,9 \pm 0,6$ & Same \\
& $-80,4 \pm 0,3$ & $-79,7 \pm 0,4$ & Same \\
& $-87,8 \pm 0,3$ & $-88,0 \pm 0,2$ & Same \\
3501 & $-80,5 \pm 0,1$ & $-80,42 \pm 0,07$ & Same \\
4003 & $-73,6 \pm 0,3$ & $-73,4 \pm 0,2$ & Same \\
& $-80,0 \pm 0,2$ & $-80,3 \pm 0,1$ & Same \\
4506 & $-73,2 \pm 0,1$ & $-74,0 \pm 0,2$ & Different \\
& $-80,94 \pm 0,08$ & $-81,2 \pm 0,1$ & Different \\
4994 & $-72,0 \pm 0,2$ & $-69,04 \pm 0,06$ & Different \\
& $-86,9 \pm 0,2$ & $-86,5 \pm 0,4$ & Same \\
5511 & $-75,1 \pm 0,1$ & $-74,3 \pm 0,3$ & Different \\
6011 & $-65,96 \pm 0,04$ & $-66,42 \pm 0,05$ & Different \\
6538 & $-75,7 \pm 0,2$ & $-75,0 \pm 0,1$ & Different \\
& $-85,0 \pm 0,4$ & $-85,3 \pm 0,3$ & Same \\
7511 & $-70,28 \pm 0,02$ & $-69,32 \pm 0,06$ & Different \\
\hline & $-78,9 \pm 0,1$ & $-75,24 \pm 0,07$ & Different \\
\hline & & &
\end{tabular}

Table 2. Acoustic amplitudes at many frequencies of laser modulation system (continuance).

\begin{tabular}{llll}
\hline $\mathbf{f}(\mathbf{H z})$ & \multicolumn{2}{c}{$\bar{A} \pm \Delta A(\mathbf{d B})$} & \multirow{2}{*}{ Note } \\
\cline { 2 - 3 } & With & Without & \\
\hline 6538 & $-75,7 \pm 0,2$ & $-75,0 \pm 0,1$ & Different \\
& $-85,0 \pm 0,4$ & $-85,3 \pm 0,3$ & Same \\
6998 & $-70,28 \pm 0,02$ & $-69,32 \pm 0,06$ & Different \\
7511 & $-78,9 \pm 0,1$ & $-75,24 \pm 0,07$ & Different \\
8004 & $-69,4 \pm 0,1$ & $-69,8 \pm 0,1$ & Different \\
8511 & $-74,34 \pm 0,08$ & $-74,3 \pm 0,2$ & Same \\
9006 & $-73,3 \pm 0,1$ & $-73,54 \pm 0,02$ & Dfferent \\
9487 & $-75,44 \pm 0,08$ & $-77,4 \pm 0,1$ & Different \\
9981 & $-69,98 \pm 0,04$ & $72,54 \pm 0,05$ & Different \\
\hline
\end{tabular}

The microphone detected photoacoustic signals on several laser modulation frequencies: $1000 \mathrm{~Hz}, 2500$ $\mathrm{Hz}, 4500 \mathrm{~Hz}, 5511 \mathrm{~Hz}, 6011 \mathrm{~Hz}, 6998 \mathrm{~Hz}, 7511 \mathrm{~Hz}$, 
$8004 \mathrm{~Hz}, 9006 \mathrm{~Hz}$, and $9981 \mathrm{~Hz}$. In some of laser modulation frequencies, there are acoustic signal peaks which indicate the photoacoustic signals.

If the test results are compared, there were amplitude differences at some laser modulation frequencies. Those differences were possible due to differences in test samples so material absorption of laser light was different. Therefore, the amplitude effect of produced photoacoustic signals was different.

However, amplitude differences at two test samples did not show a definite pattern at all laser modulation frequencies. The first analysis, this was due to the selection of modulation frequency. This selection was very important, it could be that amplitude difference occurs at some modulation frequencies only. Meanwhile, other laser modulation frequencies were not suitable frequencies or optimal frequencies for both samples. Therefore, further studies are needed for laser modulation frequencies. Further analysis, this can be caused by the selection of laser and position microphone. Therefore, further research on ideal position of both to the sample is needed.

The test results also recommend that the next implementation of green laser photoelectric spectroscopy system in a particular sample would be best to choose only one laser modulation frequency which produces photoelectric signals with a significant amplitude difference. In this test, a significant amplitude difference was found in the $7551 \mathrm{~Hz}$ modulation frequency.

\section{CONCLUSIONS}

The result showed that there were not any photoacoustic signals detected in condition of without laser and with non-modulated laser. Meanwhile, in the condition of modulated laser without sample and with sample of wood, the data acquisition system detected photoacoustic signals in the range of laser modulation frequency was $1000-9981 \mathrm{~Hz}$.

\section{REFERENCES}

Amin, M.N. and Mitrayana. 2013. Design of CO2 Laser Photoacoustic Spectrometer for Testing The Postharvest Fruit Ethylene Gas Scrubber. National Seminar of Physics and Physics Education of Sebelas Maret University Surakarta. [Indonesian]

Bageshwar, D.V; Pawar, A.S; Khanvilkar V.V dan Kadam, V.J. 2010. Photoacoustic Spectroscopy and Its Applications - A Tutorial Revie. Eurasian J. Anal. Chem. 5(2): 187-203

Rakhmadi, F.A; Mitrayana, and Shalihah, Hidayatus. 2018. Design of Green Laser Modulation System Based Based on Arduino Uno Microcontroller. AIP Conference Proceedings 2014 (020149-1-020149-5; https://doi.org/10.1063/1.5054553

Rosencwaig, Allan. 1975. Photoacoustic Spectroscopy of Solids. Physics Today.

Rosencwaig, Allan dan Gersho, Allen. 1975. Theory Of The Photoacoustic Effect With Solids. J. Appl. Phys. 47, 64 (1976); doi: 10.1063/1. 
THIS PA GE INTENTIONALLY LEFT BLANK 